\title{
ORT. I 0 - Experimental strategy to identify and validate membrane proteins as a diagnostic and therapeutic target for breast cancer
}

\author{
Julia Badaró Mendonça ${ }^{1 *}$; Mariana Caldas Waghabi'; Tatiana Martins Tilli². \\ 1Fiocruz/IOC; \\ 2Fiocruz/CDTS.
}

Introduction: The main limitations of the efficacy of currently used drugs for the treatment of cancer include systemic toxicity, drug resistance and debilitating side effects. Effective solutions to overcome these limitations are (i) membrane proteins to address the drug delivery system encapsulated in second generation biocompatible nanoparticles, and (ii) targeted monoclonal antibodies against specific targets of tumor cells. In addition, tumor-specific membrane proteins may assist in molecular diagnosis as a tumor biomarker.

Objective: In this context, this project outlined a strategy for the optimal selection of membrane proteins in tumors with a focus on the development of breast cancer therapy and diagnosis.

Methodology: Our strategy is to access the TCGA (The Cancer Genome Atlas) using transcriptome data from tumor and non-tumor human breast tissue; and distinct healthy tissues such as bladder, lung, pancreas, uterus, cervix, and colon. By this strategy, it was possible to identify membrane proteins with increased levels of expression in tumor tissue as compared to healthy tissues.

Results: Accordingly, such proteins are expected to be suitable targets for therapy with a lower rate of adverse effects and greater therapeutic efficacy. A list of seven target proteins (patents being drafted) has been identified from this inference for 96 breast tumor patients including the different molecular subtypes; Luminal A, Luminal B, HER2+ and Triple Negative. Protein validation was performed using a cohort of 1102 breast cancer patients, including clinical and pathological data. For the in vitro validations, we analyzed the expression profile of four membrane proteins by the immunofluorescence assay in breast tumor lines (MDA-MB-231, T47D, HCC1954) compared to a non-tumoral breast line (MCF10A), which were differentially expressed. In addition, the proteins identified demonstrated high specificity and sensitivity according to the ROC curve, demonstrating the potential of these membrane proteins as a biomarker and potential target for breast cancer.

Conclusion: The next step consists of the experimental validation by tissue microarray assay, including samples from breast cancer, normal and metastatic patient tissue. Consequently, we hope to validate the expression of these proteins to be appropriate targets in increasing the therapeutic efficiency and lower rate of side effects.

Keywords: Breast Cancer; Therapy; Tumor Biomarker 